\title{
Pulsar as barycenter coordinate clock
}

\author{
Yuri P. Ilyasov ${ }^{1}$, S. M. Kopeikin ${ }^{2}$, Mikhail V. Sazhin ${ }^{3}$, \\ and Vladimir E. Zharov ${ }^{3}$ \\ ${ }^{1}$ Pushchino Radio Astronomical Observatory, Lebedev Physical Institute, \\ Leninskii prosp 53, RU-142290 Puschino, Russian Federation \\ email: ilyasov@prao.psn.ru \\ ${ }^{2}$ Department of Physics and Astronomy, University of Missouri, \\ 322 Physics Building, Columbia, MO 65211, USA \\ ${ }^{3}$ Sternberg Astronomical Institut, Moscow State University, \\ Universitetskij pr., 13, RU-119992 Moscow, Russian Federation \\ email: snn,zharov@sai.msu.ru
}

\begin{abstract}
Pulsars can be considered as very precise clocks if they are observed from the barycenter of the Solar system. Pulsar Timing Array (PTA) can be used to establish new astronomical reference frame which describes both space and time properties. Among these arrays most remarkable are the Parks Pulsar Timing Array (PPTA) and Kalyazin Pulsar Timing Array (KPTA).
\end{abstract}

Keywords. pulsars: general, time, standards

The pulsar motion in space has to be taken into account. The pulsar proper motion is determined by timing technique, and transversal velocity is observed and measured by VLBI. It is impossible to measure pulsar radial velocity because there are not known spectral lines in pulsar spectra. The radial component of velocity of pulsar can not be determined by timing as well due to fact that radial velocity, radial acceleration are renormalized in pulsar period and its derivatives due to the Doppler shift. The Shapiro time delay, which is generated by average gravitational field of our Galaxy, affects the pulsar clock, as well.

Stability of the set of pulsar clocks can be satisfactory when the mutual motion of a pulsar and the observer (referred to the barycenter of the Solar system) can be considered as linear. It is evident, that pulsars having large acceleration can be excluded from PTA, for instance, pulsars belonging to Globular clusters. It is why we have to know the relative acceleration pulsar versus observer.

From ten years of observations of the pulsars at Kalyazin one can say that intrinsic and extrinsic (interstellar medium) instabilities for the best millisecond reference pulsars are about $10^{-14}$. One can expect that instability of pulsar time scale during 50-100 years will be about $10^{-18}$, and secular aberration of the Solar system should be taken into account on the level $-10^{-20} / \mathrm{yr}$.

Each of effects which affect pulsar long-term stability will be very important astronomical discovery. 